\title{
Commentary on the Article 'Healing of Amyotrophic Lateral Sclerosis: A Case Report' by Mangelsdorf et al.
}

\begin{abstract}
Johannes Naumann
Institut für Infektionsprävention und Krankenhaushygiene Interdisziplinäres Forschungs- und Behandlungszentrum (IBF) Balneologie, Universitätsklinikum Freiburg, Bad Krozingen, Germany
\end{abstract}

As a colleague of Dr. Mutter, I am very happy to see that he published a case report on one of his successful treatments in very severe diseases, particularly amyotrophic lateral sclerosis (ALS). Thereby, a larger community can become acquainted with this socalled 'causal therapy'.

It is absolutely impressive that a motor neuron disease was not only stopped in its natural progression, but has been completely reversed into recovery. One strength of the article is that the possible differential diagnoses were very well discussed and ruled out, as in nearly every cured ALS case, the primary diagnosis is doubted afterwards.

Here, the patient was diagnosed with 'progressive muscular atrophy' (PMA), a special subtype of ALS. In some cases, this disease shows a very diverse, in some a very benign clinical course [1]. But this patient had also signs of an upper motor neuron deficit in the Musculus masseter and showed a complete remission. This is very rare, and as the improvement started with the beginning of the therapy, a causal relation with the treatment is probable, as the authors state correctly from my point of view.

Surprising to me was the very fast improvement already after the start of the dental, nutritional, and micronutritional treatment, even before the initiation of chelation therapy, the removal of titanium screws as well as tattoos, and psychotherapy.
In addition, given the relatively long time lapses between the removal of the amalgam fillings in 1999, the herpes zoster-associated encephalitis in 2004, and the beginning of the symptoms in 2012, 1 year after a divorce, it does not seem very probable to me that mercury was the main cause of PMA or ALS in this patient, but perhaps the osteitis in the jaw in combination with already partially damaged nerves and other distressing factors.

As in any complex therapy, the question, which part of the therapy was crucial for the good result, remains unanswered.

It would have been helpful if more urinary mercury measurements after the treatment with 2,3-dimercapto-1-propanesulfonic acid (DMPS) would have been reported, since, according to my experience, laboratory errors occur sometimes and may well have been the cause of the very high $\mathrm{Hg}$-concentration after the seventh DMPS treatment. But this is only a small point of criticism in an otherwise good clinical documentation.

I hope, the authors will publish more successful treatments of ALS cases by Joachim Mutter, as this would show even more clearly that this case was not a spontaneous healing, but the result of a complex causal treatment.

\section{References}

1 Liewluck T, Saperstein DS: Progressive muscular atrophy. Neurol Clin 2015;33:761-773.

\section{KARGER}

() 2017 S. Karger GmbH, Freiburg
Institut für Infektionsprävention und Krankenhaushygiene Interdisziplinäres Forschungs- und Behandlungszentrum (IBF) Balneologie Universitätsklinikum Freiburg

Herbert-Hellmann-Allee 12, 79189 Bad Krozingen, Germany

johannes.naumann@uniklinik-freiburg.de 